\title{
New results on Caputo fractional-order neutral differential inclusions without compactness
}

\author{
Manar A. Alqudah' ${ }^{1}$, C. Ravichandran², Thabet Abdeljawad ${ }^{3,4,5^{*}}$ (1) and N. Valliammal ${ }^{6}$
}

\section{"Correspondence:}

tabdeljawad@psu.edu.sa

${ }^{3}$ Department of Mathematics and

General Sciences, Prince Sultan

University, Riyadh, Saudi Arabia

${ }^{4}$ Department of Medical Research,

China Medical University, Taichung, Taiwan

Full list of author information is available at the end of the article.

\begin{abstract}
This article deals with existence results of Caputo fractional neutral inclusions without compactness in Banach space using weak topology. In fact, for weakly sequentially closed maps we apply fixed point theorems to obtain the existence of the solution. Furthermore, the results are manifested for fractional neutral system held by nonlocal conditions. To justify the application of the reported results an illustration is presented.
\end{abstract}

MSC: 34A08; 93B05; 34G20; 34G25; 47H10; 47H2O

Keywords: Existence; Fractional differential inclusions; Neutral equations; Semigroup theory; Multivalued map

\section{Introduction}

The dynamical behavior of real life phenomenon are summarized by essential tools such as fractional differential equations (FDEs) in a precise manner. This aspect is the main convenience of derivatives with fractional-order versus integer-order models. FDEs and inclusions have obtained many interest for their applications in different fields, such as engineering, physics, mechanics, and mathematical modelling, because they are more practi$\mathrm{cal}$ and realistic to describe many natural phenomena. Compared with ordinary and partial differential systems, fractional differential systems have the strong prospect to modulate the real time issues with high efficiency. The goal of analyzing fractional differential systems for the above, major analysis $[2-4,6,7,12,23,25-28,30-32,38,40,42,47,48,56-$ 58 ] had been carried out. El-Sayed and Ibrahim in [24]. were the first who considered fractional differential inclusions.

Furthermore, differential inclusions are used to model many realistic problems, arising from optimal control, economics, and so on. Recently, by using various techniques, the mild solutions together with other issues for different types of nonlinear fractional evolution inclusions have been studied in $[1,5,13-17,21,22,33,43-45,49-52,54,55,59]$.

We recall that several techniques and noncompact measures are used to achieve the outcome of the differential systems. Most of these papers, assumed the compactness of the semi group or alternatively a compactness condition on the nonlinear part (generally a measure of noncompactness). In [46], Ravichandran et al. analyzed the controllabil-

(c) The Author(s) 2019. This article is licensed under a Creative Commons Attribution 4.0 International License, which permits use, sharing, adaptation, distribution and reproduction in any medium or format, as long as you give appropriate credit to the original author(s) and the source, provide a link to the Creative Commons licence, and indicate if changes were made. The images or other third party material in this article are included in the article's Creative Commons licence, unless indicated otherwise in a credit line to the material. If material is not included in the article's Creative Commons licence and your intended use is not permitted by statutory regulation or exceeds the permitted use, you will need to obtain permission directly from the copyright holder. To view a copy of this licence, visit http://creativecommons.org/licenses/by/4.0/. 
ity of impulsive fractional integro-differential systems utilizing a contraction principle. Li [33] studied the controllability results for neutral impulsive inclusion systems using the Dhage fixed point theorem. The controllability for evolution inclusions without compactness was studied by Benedetti et al. [15]. To the best of our knowledge, the existence of mild solutions of Caputo fractional neutral differential inclusions without compactness has not been studied and this is the main motivation of this work, is to prove the existence of Caputo fractional neutral differential inclusions with weak topology, and without compactness. We investigate the following Caputo fractional neutral inclusion in Banach space:

$$
\begin{aligned}
& { }^{\mathrm{C}} D_{u}^{q}[z(u)-h(u, z(u))] \in \mathscr{A} z(u)+\mathscr{H}(u, z(u)), \quad \text { a.e. } u \in[0, b], 0<q \leq 1, \\
& z(0)=z_{0},
\end{aligned}
$$

where $b$ is positive in nature, $\mathscr{A}$ represents the infinitesimal generator of a $C_{0}$-strongly continuous semigroup $T(u), u \geq 0$, defined from $\mathscr{A}: D(\mathscr{A}) \rightarrow \mathbb{Y}$. Besides, $z(\cdot)$ assumes values in the Banach space $\mathbb{Y}, z_{0}$ is for an element of $\mathbb{Y}, \mathscr{H}:[0, b] \times \mathbb{Y} \multimap \mathbb{Y}$ denotes a multivalued map, $h:[0, b] \times \mathbb{Y} \rightarrow \mathbb{Y}$ is equicontinuous and bounded.

The aim of this paper is to derive some sufficient conditions for the existence of neutral differential inclusions in Banach space. Furthermore, we expand the result to get the conditions for neutral differential inclusions with nonlocal conditions. In this paper another procedure is considered, it utilize the weak topology of the state space.

Considering the importance of modeling of crisis phenomena, one may extend the analysis to the existence of solutions for a three step crisis integro-differential equation. Also in order to rise the applicability of the fractional calculus, many researchers assumed a new type of fractional derivatives with different kernels. By exploiting it, one can examine the existence of solutions for high-order fractional differential equations using the CaputoFabrizio derivative [8-11, 36, 37].

The layout of this artical is as follows: The preliminaries and notations are listed in Sect. 2. The existence results are discussed in Sect. 3, and in Sect. 4 we investigate the same fractional system supported by nonlocal conditions. An illustration is offered to enhance the abstract technique.

\section{Basic tools}

Here, we present a few fundamental facts on fractional theory and theorems in order to use them in the manuscript.

Let $\mathbb{Y}$ possessing $\|\cdot\|$. For some constant $\mathscr{M}_{1}>0$ provided $\sup _{u \in[0, b]}\|T(u)\| \leq \mathscr{M}_{1}$. Let $(\mathbb{Y},\|\cdot\|)$ be a reflexive Banach space and $\mathbb{Y}_{w}$ denotes $\mathbb{Y}$ equipped with the weak topology. $\mathfrak{C}([0, b] ; \mathbb{Y})$ refers the Banach space of all continuous functions from $[0, b]$ into $\mathbb{Y}$. For $\mathscr{D} \subset$ $\mathbb{Y}, \overline{\mathscr{D}}^{w}$ signifies the weak closure of $\mathscr{D}$. Moreover, the bounded subset $\mathscr{D}$ of a reflexive Banach space $\mathbb{Y}$ is weakly relatively compact. Let us express by $\|\cdot\|_{p}$ both the $L^{p}([0, b] ; \mathbb{Y})$ norm and the $L^{p}([0, b] ; \mathbb{R})$-norm and by $\|\cdot\|_{0}$ the $\mathfrak{C}([0, b] ; \mathbb{Y})$-norm. We evoke (see [18], Theorem 4.3) that the sequence $\left\{\chi_{n}\right\} \subset \mathfrak{C}([0, b] ; \mathbb{Y})$ converges weakly to $\chi \in \mathfrak{C}([0, b] ; \mathbb{Y})$ if and only if

(i) there exists $M>0$ such that, for every $m \in \mathbb{N}$ and $u \in[0, b],\left\|\chi_{m}(u)\right\| \leq M$; 
(ii) $\chi_{m}(u) \rightarrow \chi(u)$, for every $u \in[0, b]$.

In addition to that, we state some results that will be utilized further as a part of this manuscript.

Theorem 2.1 (Donal O'Regan fixed point theorem [39]) Let F be a metrizable locally convex linear topological space and $U$ be a weakly compact, convex subset of $F$ and $\mathbb{C}(U)$ the family of nonempty closed, convex subsets of $U$. If $G: U \rightarrow \mathbb{C}(U)$ possesses weakly sequentially closed graph then $G$ admits a fixed point.

Theorem 2.2 ([29]) Let $\Psi$ be a subset of the Banach space $\mathbb{Y}$ then the subsequent affirmations are equivalent, namely:

(i) $\Psi$ is relatively weakly compact;

(ii) $\Psi$ is relatively weakly sequentially compact.

Remark 2.1 ([29]) Let $\Psi$ be a subset of the Banach space $\mathbb{Y}$, then the subsequent affirmations are equivalent:

(a) $\Psi$ is weakly compact;

(b) $\Psi$ is weakly sequentially compact.

Theorem 2.3 (Krein-Smulian theorem [23, p. 434]) The convex hull of a weakly compact set in a Banach space $\mathbb{Y}$ is weakly compact.

Theorem 2.4 (Pettis measurability theorem [41]) Let $(E, \Sigma)$ be a measurable space, $\mathbb{Y}$ be a separable Banach space. Then $f: E \longrightarrow \mathbb{Y}$ is measurable if and only if, for every $e \in E^{\prime}$, the function o $f: E \longrightarrow \mathbb{R}$ is measurable with respect to $\sum$ and the Borel $\sigma$-algebra in $\mathbb{R}$.

Now we summarize the subsequent interpretations [7, 31, 32, 38, 42].

Definition 2.1 The form of the fractional integral for $f$ is

$$
I^{\alpha} g(p)=\frac{1}{\Gamma(\alpha)} \int_{0}^{p} \frac{g(w)}{(p-w)^{1-\alpha}} d w, \quad p>0, \alpha>0
$$

as the right hand-side is point-wise on $[0, \infty)$ and $\Gamma(\alpha)=\int_{0}^{\infty} p^{\alpha-1} e^{-p} d p$.

Definition 2.2 ([31]) The form of the R-L fractional derivative for $g:[0, \infty) \rightarrow \mathbb{R}$ is characterized by

$$
{ }^{(\mathrm{R}-\mathrm{L})} D_{0+}^{\alpha} g(p)=\frac{1}{\Gamma(m-\alpha)}\left(\frac{d}{d p}\right)^{m} \int_{0}^{p}(p-w)^{m-\alpha-1} g(w) d w, \quad p>0, m-1<\alpha<m,
$$

such that the function $g(p)$ posses absolutely continuous derivative up to $(m-1)$.

Definition 2.3 ([31]) The expression of the Caputo derivative for $g:[0, \infty) \rightarrow \mathbb{R}$ is

$$
{ }^{\mathrm{C}} D^{\alpha} g(p)={ }^{(\mathrm{R}-\mathrm{L})} D^{\alpha}\left(g(p)-\sum_{k=0}^{m-1} \frac{p^{k}}{k !} g^{(k)}(0)\right), \quad p>0, m-1<\alpha<m
$$


Remark 2.2 (i) If $g(p) \in C^{m}[0, \infty)$, then

$$
{ }^{\mathrm{C}} D^{\alpha} g(p)=\frac{1}{\Gamma(m-\alpha)} \int_{0}^{p} \frac{g^{(m)}(w)}{(p-w)^{\alpha+1-m}} d w=I^{m-\alpha} g^{(m)}(p), \quad p>0, m-1<\alpha<m .
$$

\section{Existence results}

Below, we demonstrate some sufficient conditions for the existence of (1.1)-(1.2) coupled with weak topology.

$\left(\mathbf{H}_{\mathbf{1}}\right)$ For $\{T(u)\}_{u \geq 0}$ in $\mathbb{Y}$, there is a constant $\mathscr{M}_{1} \geq 1$ fulfilling $\sup _{u \in[0, b]}\|T(u)\| \leq \mathscr{M}_{1}$.

Additionally, we require that the multivalued nonlinearity function $\mathscr{H}:[0, b] \times$ $\mathbb{Y} \multimap \mathbb{Y}$ possess nonempty convex and weakly compact values.

$\left(\mathbf{H}_{2}\right)$ For all $z \in \mathbb{Y}$, the multivalued function $\mathscr{H}(\cdot, z):[0, b] \multimap \mathbb{Y}$ has a measurable selection.

$\left(\mathbf{H}_{3}\right) \mathscr{H}(u, \cdot): \mathbb{Y} \multimap \mathbb{Y}$ is weakly sequentially closed for almost everywhere $u$ in $[0, b]$.

$\left(\mathbf{H}_{4}\right)$ For a real valued function $h:[0, b] \times \mathbb{Y} \rightarrow \mathbb{Y}$, for all $u>0$ and some constant $\mathfrak{M}_{h}>0$ we have $\|h(u, \cdot)\| \leq \mathfrak{M}_{h}$.

$\left(\mathbf{H}_{5}\right)$ For $\kappa_{1} \in(0, q)$, for every $r>0$ and a function $\delta_{r} \in L^{\frac{1}{\kappa_{1}}}\left([0, b] ; \mathbb{R}_{+}\right)$as for each $d \in \mathbb{Y}$, $\|d\| \leq r$ :

$$
\|h(u, d)\|=\sup \{\|z\|: z \in h(u, d)\} \leq \delta_{r}(u)
$$

for almost everywhere $u \in[0, b]$.

( $\left.\mathbf{H}_{\mathbf{6}}\right)$ For $\kappa_{1} \in(0, q)$, for every $r>0$ and a function $\mu_{r} \in L^{\frac{1}{\kappa_{1}}}\left([0, b] ; \mathbb{R}_{+}\right)$as for each $d \in \mathbb{Y}$, $\|d\| \leq r$

$$
\|\mathscr{H}(u, d)\|=\sup \{\|z\|: z \in \mathscr{H}(u, d)\} \leq \mu_{r}(u)
$$

for almost everywhere $u \in[0, b]$.

In connection with the above consideration, we determine the solution of (1.1)-(1.2).

Definition 3.1 ([56]) $z:[0, b] \rightarrow \mathbb{Y}$ is a mild solution of (1.1)-(1.2) if the accompanying recognize $z(0)=z_{0}$ and there is $\chi \in L^{\frac{1}{\kappa_{1}}}([0, b] ; \mathbb{Y})$ provided $\chi(u) \in \mathscr{H}(u, z(u))$ on $u \in[0, b]$ and $z$ fulfills

$$
\begin{aligned}
z(u)= & \mathbb{T}(u)\left[z_{0}-h\left(0, z_{0}\right)\right]+h(u, z(u))+\int_{0}^{u}(u-w)^{q-1} \mathscr{A} \mathbb{S}(u-w) h(w, z(w)) d w \\
& +\int_{0}^{u}(u-w)^{q-1} \mathbb{S}(u-w) \chi(w) d w, \quad u \in[0, b],
\end{aligned}
$$

where

$$
\mathbb{T}(u)=\int_{0}^{\infty} \xi_{q}(\theta) T\left(u^{q} \theta\right) d \theta, \quad \mathbb{S}(u)=q \int_{0}^{\infty} \theta \xi_{q}(\theta) T\left(u^{q} \theta\right) d \theta,
$$

and, for $\theta \in(0, \infty)$,

$$
\xi_{q}(\theta)=\frac{1}{q} \theta^{-1-\frac{1}{q}} \bar{w}_{q}\left(\theta^{-\frac{1}{q}}\right) \geq 0,
$$




$$
\begin{aligned}
& \bar{w}_{q}(\theta)=\frac{1}{\pi} \sum_{n=1}^{\infty}(-1)^{n-1} \theta^{-n q-1} \frac{\Gamma(n q+1)}{n !} \sin (n \pi q), \\
& \int_{0}^{\infty} \xi_{q}(\theta) d \theta=1 .
\end{aligned}
$$

Remark 3.1 Obviously, for $v \in[0,1]$,

$$
\int_{0}^{\infty} \theta^{v} \xi_{q}(\theta) d \theta=\int_{0}^{\infty} \theta^{-q v} \bar{w}_{q}(\theta) d \theta=\frac{\Gamma(1+v)}{\Gamma(1+q v)} .
$$

Lemma 3.1 (See [56]) $\mathbb{T}$ and $\mathbb{S}$ obey the subsequent assertions:

(i) For a constant $\mathscr{M}_{2} \geq 1$, for any $z \in \mathbb{Y}$, fixed $u \geq 0$ and for the bounded linear operators $\mathbb{T}$ and $\mathbb{S}$ we have

$$
\begin{aligned}
& \|\mathbb{T}(u) z\| \leq \mathscr{M}_{1}\|z\| \quad \text { and } \quad\|\mathbb{S}(u) z\| \leq \frac{q \mathscr{M}_{1}}{\Gamma(1+q)}\|z\|, \\
& \|\mathscr{A} \mathbb{S}(u) z\| \leq \frac{q \mathscr{M}_{1} \mathscr{M}_{2}}{\Gamma(1+q)}\|z\| .
\end{aligned}
$$

(ii) The operators $\{\mathbb{T}(u), u \geq 0\}$ and $\{\mathbb{S}(u), u \geq 0\}$ are strongly continuous.

Construct the set $\Upsilon_{\wp}$, for given $\wp \in \mathfrak{C}([0, b] ; \mathbb{Y})$ as $\Upsilon_{\wp}=\left\{\chi \in L^{\frac{1}{\kappa_{1}}}([0, b] ; \mathbb{Y}): \chi(u) \in\right.$ $\mathscr{H}(u, \wp(u))$ for almost everywhere $u \in[0, b]\} . \Upsilon_{\wp}$ is nonempty as the next Proposition 3.1 mentions.

Proposition 3.1 (See $[15,59]$ ) Let us assume that a multivalued map $\mathscr{H}:[0, b] \times \mathbb{Y} \multimap \mathbb{Y}$ obeys $\left(\mathbf{H}_{2}\right)-\left(\mathbf{H}_{\mathbf{6}}\right)$, the set $\Upsilon_{\wp}$ is nonempty for any $\wp \in \mathfrak{C}([0, b] ; \mathbb{Y})$.

We define the operator $\Lambda: \mathfrak{C}([0, b] ; \mathbb{Y}) \multimap \mathfrak{C}([0, b] ; \mathbb{Y})$ by

$$
\begin{aligned}
\Lambda(\wp)= & \left\{z \in \mathfrak{C}([0, b] ; \mathbb{Y}): z(u)=\mathbb{T}(u)\left(z_{0}-h\left(0, z_{0}\right)\right)+h(u, \wp(u))\right. \\
& \left.+S_{1}(z)(u)+S_{2} \chi(u), \chi \in \Upsilon_{\wp}\right\},
\end{aligned}
$$

where

$$
\begin{aligned}
& S_{1}: \mathfrak{C}([0, b] ; \mathbb{Y}) \rightarrow \mathfrak{C}([0, b] ; \mathbb{Y}), \\
& S_{1}(z)=\int_{0}^{u}(u-w)^{q-1} \mathscr{A} \mathbb{S}(u-w) h(w, \wp(w)) d w,
\end{aligned}
$$

and

$$
\begin{aligned}
& S_{2}: L^{\frac{1}{\kappa_{1}}}([0, b] ; \mathbb{Y}) \rightarrow \mathfrak{C}([0, b] ; \mathbb{Y}), \\
& S_{2}(\chi)=\int_{0}^{u}(u-w)^{q-1} \mathbb{S}(u-w) \chi(w) d w .
\end{aligned}
$$

At first, we show that $S_{1}$ and $S_{2}$ are continuous.

For any $z_{n}, z \in \mathfrak{C}([0, b] ; \mathbb{Y})$ and $z_{n} \rightarrow z(n \rightarrow \infty)$, using $\left(\mathbf{H}_{5}\right)$, for every $u \in[0, b]$, we get

$$
(u-w)^{q-1}\left\|z_{n}(w)-z(w)\right\| \leq 2(u-w)^{q-1} \mu_{r}(w), \quad \text { almost everywhere } w \in[0, u) .
$$


Also for any $\chi_{n}, \chi \in L^{\frac{1}{\kappa_{1}}}([0, b] ; \mathbb{Y})$ and $\chi_{n} \rightarrow \chi(n \rightarrow \infty)$, using $\left(\mathbf{H}_{\mathbf{6}}\right)$, we can have, for every $u \in[0, b]$,

$$
(u-w)^{q-1}\left\|\chi_{n}(w)-\chi(w)\right\| \leq 2(u-w)^{q-1} \delta_{r}(w), \quad \text { almost everywhere } w \in[0, u) .
$$

Moreover, the functions

$$
\int_{0}^{u}(u-w)^{q-1} \mu_{r}(w) d w=\left[\left(\frac{1-\kappa_{1}}{q-\kappa_{1}}\right) b^{\frac{q-\kappa_{1}}{1-\kappa_{1}}}\right]^{1-\kappa_{1}}\left\|\mu_{r}\right\|_{\frac{1}{\kappa_{1}}}
$$

and

$$
\int_{0}^{u}(u-w)^{q-1} \delta_{r}(w) d w=\left[\left(\frac{1-\kappa_{1}}{q-\kappa_{1}}\right) b^{\frac{q-\kappa_{1}}{1-\kappa_{1}}}\right]^{1-\kappa_{1}}\left\|\delta_{r}\right\|_{\frac{1}{\kappa_{1}}}
$$

becomes integrable for $u \in[0, b]$. Taking into account the Lebesgue theorem, we conclude, as $n \rightarrow \infty$,

$$
\int_{0}^{u}(u-w)^{q-1}\left\|z_{n}(w)-z(w)\right\| d w \rightarrow 0 \quad \text { and } \quad \int_{0}^{u}(u-w)^{q-1}\left\|\chi_{n}(w)-\chi(w)\right\| d w \rightarrow 0 .
$$

Therefore

$$
\begin{aligned}
\left\|S_{1}\left(z_{n}\right)-S_{1}(z)\right\| & \leq\left\|\int_{0}^{u}(u-w)^{q-1} \mathscr{A} \mathbb{S}(u-w)\left(z_{n}(w)-z(w)\right) d w\right\| \\
& \leq \frac{q \mathscr{M}_{1} \mathscr{M}_{2}}{\Gamma(1+q)} \int_{0}^{u}(u-w)^{q-1}\left\|z_{n}(w)-z(w)\right\| d w \rightarrow 0, \quad \text { as } n \rightarrow \infty, \\
\left\|S_{2}\left(\chi_{n}\right)-S_{2}(\chi)\right\| & \leq\left\|\int_{0}^{u}(u-w)^{q-1} \mathbb{S}(u-w)\left(\chi_{n}(w)-\chi(w)\right) d w\right\| \\
& \leq \frac{q \mathscr{M}_{1}}{\Gamma(1+q)} \int_{0}^{u}(u-w)^{q-1}\left\|\chi_{n}(w)-\chi(w)\right\| d w \rightarrow 0, \quad \text { as } n \rightarrow \infty .
\end{aligned}
$$

It indicates that the operators $S_{1}$ and $S_{2}$ are continuous.

For $n \in \mathbb{N}, \Phi_{n}$, the closed ball of radius $n$ in $\mathfrak{C}([0, b] ; \mathbb{Y})$ described by $\Lambda_{n}=\Lambda \mid \Phi_{n}: \Phi_{n} \multimap$ $\mathfrak{C}([0, b] ; \mathbb{Y})$, the limitation of $\Lambda$ on $\Phi_{n}$. Next we illustrate the qualities of $\Lambda_{n}$.

Proposition 3.2 $\Lambda_{n}$ possess a weakly sequentially closed graph.

Proof Let a sequence $\left\{\wp_{m}\right\} \subset \Phi_{n},\left\{z_{m}\right\} \subset \mathfrak{C}([0, b] ; \mathbb{Y})$ obeying $z_{m} \subset \Lambda_{n}\left(\wp_{m}\right)$, for all $m$ and $\wp_{m} \rightarrow \wp, z_{m} \rightarrow z$ in $\mathfrak{C}([0, b] ; \mathbb{Y})$; we claim $z \in \Lambda_{n}(\wp)$.

Since $\wp_{m} \in \Phi_{n}$, for each $m$ and $\wp_{m}(u) \rightarrow \wp(u)$, for every $u \in[0, b]$, we conclude $\|\wp(u)\| \leq$ $\lim _{\text {inf }_{m} \rightarrow \infty}\left\|\wp_{m}(u)\right\| \leq n$, for all $u$ (see [19]). By $z_{m} \in \Lambda_{n}\left(p_{m}\right)$, there is a sequence $\left\{\chi_{m}\right\}, \chi_{m} \in$ $\Upsilon_{\wp m}$, provided for all $u \in[0, b]$, we get

$$
\begin{aligned}
z_{m}(u)= & \mathbb{T}(u)\left(z_{0}-h\left(0, z_{0}\right)\right)+h\left(u, \wp_{m}(u)\right)+\int_{0}^{u}(u-w)^{q-1} \mathscr{A} \mathbb{S}(u-w) h\left(w, \wp_{m}(w)\right) d w \\
& +\int_{0}^{u}(u-w)^{q-1} \mathbb{S}(u-w) \chi_{m}(w) d w .
\end{aligned}
$$


By reference to $\left(\mathbf{H}_{\mathbf{6}}\right),\left\|\chi_{m}(u)\right\| \leq \mu_{n}(u)$, for almost everywhere $u$ and every $m$. It means that $\left\{\chi_{m}\right\}$ is bounded, uniformly integrable and $\left\{\chi_{m}(u)\right\}$ is bounded in $\mathbb{Y}$ for almost everywhere $u \in[0, b]$. By the Dunford-Pettis theorem [13], we can conclude that there exist a subsequence, represented as the sequence, and functions $g_{1}, g_{2}$ provided $z_{m} \rightarrow g_{1}$ in $\mathfrak{C}([0, b] ; \mathbb{Y})$ and $\chi_{m} \rightarrow g_{2}$ in $L^{\frac{1}{\kappa_{1}}}([0, b] ; \mathbb{Y})$.

Therefore, we have $S_{1} z_{m} \rightarrow S_{1} g_{1}$ and $S_{2} \chi_{m} \rightarrow S_{2} g_{2}$. In this connection, let the linear continuous operator $e^{\prime}: \mathbb{Y} \rightarrow \mathbb{R}$. The operators $S_{1}$ and $S_{2}$ are linear and continuous, therefore we have

$$
g_{1} \rightarrow e^{\prime}\left(S_{1} g_{1}\right)(u), \quad g_{1} \in \mathfrak{C}([0, b] ; \mathbb{Y})
$$

is linear continuous operator on $\mathfrak{C}([0, b] ; \mathbb{Y})$ to $\mathbb{R}$ for every $u \in[0, b]$. Also,

$$
g_{2} \rightarrow e^{\prime}\left(S_{2} g_{2}\right)(u), \quad g_{2} \in L^{\frac{1}{\kappa_{1}}}([0, b] ; \mathbb{Y})
$$

is linear continuous operator on $L^{\frac{1}{\kappa_{1}}}([0, b] ; \mathbb{Y})$ to $\mathbb{R}$ for every $u \in[0, b]$. By weak convergence, we get

$$
\begin{gathered}
e^{\prime}\left(\int_{0}^{u}(u-w)^{q-1} \mathscr{A} \mathbb{S}(u-w) h\left(w, \wp_{m}(w)\right) d w+\int_{0}^{u}(u-w)^{q-1} \mathbb{S}(u-w) \chi_{m}(w) d w\right) \\
\rightarrow e^{\prime}\left(\int_{0}^{u}(u-w)^{q-1} \mathscr{A} \mathbb{S}(u-w) g_{1}(w) d w+\int_{0}^{u}(u-w)^{q-1} \mathbb{S}(u-w) g_{2}(w) d w\right) .
\end{gathered}
$$

Therefore

$$
\begin{aligned}
z_{m}(u) \rightarrow & \mathbb{T}(u)\left(z_{0}-h\left(0, z_{0}\right)\right)+h\left(u, \wp_{m}(u)\right)+\int_{0}^{u}(u-w)^{q-1} \mathscr{A} \mathbb{S}(u-w) g_{1}(w) d w \\
& +\int_{0}^{u}(u-w)^{q-1} \mathbb{S}(u-w) g_{2}(w) d w \\
= & z_{0}(u)
\end{aligned}
$$

for every $u \in[0, b]$. This indicates that $z_{0}(u)=z(u)$, for all $u \in[0, b]$. Hence by Proposition 3.1, $g_{2}(u) \in \mathscr{H}(u, \wp(u))$, for almost everywhere $u \in[0, b]$.

Proposition 3.3 $\Lambda_{n}$ is weakly compact.

Proof At first, we show that $\Lambda_{n}\left(\Phi_{n}\right)$ is relatively weakly sequentially compact.

Let us consider $\wp_{m} \in \Phi_{n}$ and $z_{m} \subset \mathfrak{C}([0, b] ; \mathbb{Y})$ such that $z_{m} \in \Lambda_{n}\left(\wp_{m}\right)$ for all $m$. For $\Lambda_{n}$, there exists a sequence $\left\{\chi_{m}\right\}, \chi_{m} \in \Upsilon_{\wp m}$, provided that

$$
\begin{aligned}
z_{m}(u)= & \mathbb{T}(u)\left(z_{0}-h\left(0, z_{0}\right)\right)+h\left(u, \wp_{m}(u)\right)+\int_{0}^{u}(u-w)^{q-1} \mathscr{A} \mathbb{S}(u-w) h\left(w, \wp_{m}(w)\right) d w \\
& +\int_{0}^{u}(u-w)^{q-1} \mathbb{S}(u-w) \chi_{m}(w) d w
\end{aligned}
$$

for every $u \in[0, b]$. Therefore, by Proposition 3.2, there exist a subsequence, represented by the sequence, and functions $g_{1}, g_{2}$ provided $z_{m} \rightarrow g_{1}$ in $\mathfrak{C}([0, b] ; \mathbb{Y})$ and $\chi_{m} \rightarrow g_{2}$ in 
$L^{\frac{1}{\kappa_{1}}}([0, b] ; \mathbb{Y})$. Hence

$$
\begin{aligned}
z_{m}(u) \rightarrow & l(u) \\
= & \mathbb{T}\left(z_{0}-h\left(0, z_{0}\right)\right)+h\left(u, \wp_{m}(u)\right)+\int_{0}^{u}(u-w)^{q-1} \mathscr{A} \mathbb{S}(u-w) g_{1}(w) d w \\
& +\int_{0}^{u}(u-w)^{q-1} \mathbb{S}(u-w) g_{2}(w) d w .
\end{aligned}
$$

Furthermore, by the nature of weak convergence of $\chi_{m}$, by $\left(\mathbf{H}_{\mathbf{1}}\right)$, we have

$$
\begin{aligned}
\left\|z_{m}(u)\right\| \leq & \mathscr{M}_{1}\left\|z_{0}\right\|+\delta_{r}(0)+\mathfrak{M}_{h}+\frac{\mathscr{M}_{1} \mathscr{M}_{2} q}{\Gamma(1+q)}\left[\left(\frac{1-\kappa_{1}}{q-\kappa_{1}}\right) b^{\frac{q-\kappa_{1}}{1-\kappa_{1}}}\right]^{1-\kappa_{1}}\left\|\delta_{r}\right\|_{\frac{1}{\kappa_{1}}} \\
& +\frac{\mathscr{M}_{1} q}{\Gamma(1+q)}\left[\left(\frac{1-\kappa_{1}}{q-\kappa_{1}}\right) b^{\frac{q-\kappa_{1}}{1-\kappa_{1}}}\right]^{1-\kappa_{1}}\left\|\mu_{r}\right\|_{\frac{1}{\kappa_{1}}},
\end{aligned}
$$

for all $m \in \mathbb{N}$ and $u \in[0, b]$. By utilizing the Proposition 3.2, we ensure that $z_{m} \rightarrow l$ in $\mathfrak{C}([0, b] ; \mathbb{Y})$. Thus, $\Lambda_{n}\left(\Phi_{n}\right)$ is relatively weakly compact by Theorem 2.2 .

Proposition 3.4 $\Lambda_{n}$ has convex and weakly compact values.

Proof Fixing $\wp \in \Phi_{n}$, taking into account that $\mathscr{H}$ is convex valued and the characteristics of $\mathbb{T}(u)$ and $\mathbb{S}(u)$, it implies that $\Lambda_{n}(\wp)$ is convex. By reference to Proposition 3.2 and Proposition $3.3, \Lambda_{n}(\wp)$ has weakly compact values.

Next we list out the essential outcomes of this part.

Theorem 3.1 Assuming $\left(\mathbf{H}_{\mathbf{1}}\right)-\left(\mathbf{H}_{\mathbf{6}}\right)$ hold. Suppose $\left(\mathbf{H}_{7}\right)$ for a sequence offunctions $\left\{u_{n}\right\} \in$ $L^{\frac{1}{\kappa_{1}}}\left([0, b] ; \mathbb{R}_{+}\right)$provided

$$
\sup _{\|d\| \leq n}\|\mathscr{H}(u, d)\| \leq u_{n}(u)
$$

for almost everywhere $u \in[0, b], n \in \mathbb{N}$ with

$$
\lim _{n \rightarrow \infty} \inf \frac{\left\|u_{n}\right\|_{\frac{1}{\kappa_{1}}}}{n}=0 .
$$

Then (1.1)-(1.2) recognizes at least a mild solution.

Proof We have to confirm that $\Lambda$ maps $\Phi_{n}$ into itself for $n \in \mathbb{N}$.

Assume by way of contradiction that there exist $\left\{z_{n}\right\},\left\{x_{n}\right\}$ such that $z_{n} \in \Phi_{n}, x_{n} \in \Lambda_{n}\left(z_{n}\right)$ and $x_{n} \notin \Phi_{n}$, for every $n \in \mathbb{N}$. Therefore for a sequence $\left\{\chi_{n}\right\} \subset L^{\frac{1}{\kappa_{1}}}([0, b] ; \mathbb{Y}), \chi_{n}(w) \in$ $\mathscr{H}\left(w, z_{n}(w)\right)$, we can have

$$
\begin{aligned}
x_{n}(u)= & \mathbb{T}(u)\left(z_{0}-h\left(0, z_{0}\right)\right)+h\left(u, z_{n}(u)\right)+\int_{0}^{u}(u-w)^{q-1} \mathscr{A} \mathbb{S}(u-w) h\left(w, z_{n}(w)\right) d w \\
& +\int_{0}^{u}(u-w)^{q-1} \mathbb{S}(u-w) \chi_{n}(w) d w
\end{aligned}
$$


for every $u \in[0, b]$. By Proposition 3.3, we have

$$
\begin{aligned}
n \leq & \left\|x_{n}\right\|_{0} \\
\leq & \mathscr{M}_{1}\left\|z_{0}\right\|+\delta_{r}(0)+\mathfrak{M}_{h}+\frac{\mathscr{M}_{1} \mathfrak{M}_{h} \mathscr{M}_{2} q}{\Gamma(1+q)}\left[\left(\frac{1-\kappa_{1}}{q-\kappa_{1}}\right) b^{\frac{q-\kappa_{1}}{1-\kappa_{1}}}\right]^{1-\kappa_{1}} \\
& +\frac{\mathscr{M}_{1} q}{\Gamma(1+q)}\left[\left(\frac{1-\kappa_{1}}{q-\kappa_{1}}\right) b^{\frac{q-\kappa_{1}}{1-\kappa_{1}}}\right]^{1-\kappa_{1}}\left\|u_{n}\right\|_{\frac{1}{\kappa_{1}}} .
\end{aligned}
$$

Then, for $n \in \mathbb{N}$,

$$
\begin{aligned}
1 \leq & \frac{\left\|x_{n}\right\|_{0}}{n} \\
\leq & \frac{1}{n}\left[\mathscr{M}_{1}\left\|z_{0}\right\|+\delta_{r}(0)+\mathfrak{M}_{h}+\frac{\mathscr{M}_{1} \mathfrak{M}_{h} \mathscr{M}_{2} q}{\Gamma(1+q)}\left[\left(\frac{1-\kappa_{1}}{q-\kappa_{1}}\right) b^{\frac{q-\kappa_{1}}{1-\kappa_{1}}}\right]^{1-\kappa_{1}}\right. \\
& \left.+\frac{\mathscr{M}_{1} q}{\Gamma(1+q)}\left[\left(\frac{1-\kappa_{1}}{q-\kappa_{1}}\right) b^{\frac{q-\kappa_{1}}{1-\kappa_{1}}}\right]^{1-\kappa_{1}}\left\|u_{n}\right\|_{\frac{1}{\kappa_{1}}}\right]
\end{aligned}
$$

which leads to a contradiction. Therefore $x_{n} \in \Phi_{n}$.

Now, fix $n \in \mathbb{N}$ such that $\Lambda_{n}\left(\Phi_{n}\right) \subset \Phi_{n}$. By Proposition 3.3, the set $\mathscr{V}_{n}={\overline{\Lambda_{n}\left(\Phi_{n}\right)}}^{w}$ is weakly compact. Let $\xi_{n}=\overline{\operatorname{co}}\left(\mathscr{V}_{n}\right)$, be the closed convex hull of $\mathscr{V}_{n}$. According to Theorem $2.3, \xi_{n}$ denotes a weakly compact set. In addition to that $\Lambda_{n}\left(\Phi_{n}\right) \subset \Phi_{n}$ and $\Phi_{n}$ is a closed convex set. Furthermore we have $\xi_{n} \subset \Phi_{n}$, and we have

$$
\Lambda_{n}\left(\xi_{n}\right)=\Lambda_{n}\left(\overline{\operatorname{co}}\left(\Lambda_{n}\left(\Phi_{n}\right)\right)\right) \subseteq \Lambda_{n}\left(\Phi_{n}\right) \subseteq{\overline{\Lambda_{n}\left(\Phi_{n}\right)}}^{w}=\mathscr{V}_{n} \subset \xi_{n}
$$

This shows that $\Lambda_{n}$ possesses a weakly sequentially closed graph. As a result by utilizing Theorem 2.1, we conclude that the system (1.1)-(1.2) recognizes a solution.

Remark 3.2 There exist $\alpha \in L^{\frac{1}{\kappa_{1}}}\left([0, b] ; \mathbb{R}_{+}\right)$and a nondecreasing function $\phi:[0, \infty) \rightarrow$ $[0, \infty)$ such that $\|\mathscr{H}(u, d)\| \leq \alpha(u) \phi(\|d\|)$, for almost everywhere $u \in[0, b]$ and every $d \in \mathbb{Y}$. Then the restriction (3.1) is related to

$$
\lim _{n \rightarrow \infty} \inf \frac{\phi(n)}{n}=0
$$

Theorem 3.2 Assume that $\left(\mathbf{H}_{1}\right)-\left(\mathbf{H}_{5}\right)$ holds.

$\left(\mathbf{H}_{\mathbf{8}}\right)$ There exists $\rho \in L^{\frac{1}{\kappa_{1}}}\left([0, b] ; \mathbb{R}_{+}\right)$, for almost everywhere $u \in[0, b]$, for every $d \in \mathbb{Y}$ provided

$$
\|\mathscr{H}(u, d)\| \leq \rho(u)(1+\|d\|)
$$

and

$$
\frac{\mathscr{M}_{1} q}{\Gamma(1+q)}\left[\left(\frac{1-\kappa_{1}}{q-\kappa_{1}}\right) b^{\frac{q-\kappa_{1}}{1-\kappa_{1}}}\right]^{1-\kappa_{1}}\left(\mathscr{M}_{2} \mathfrak{M}_{h}+\|\rho\|_{\frac{1}{\kappa_{1}}}\right)<1
$$

then (1.1)-(1.2) possess at least a mild solution. 
Proof By reference to Theorem 3.1, assuming that there exist $\left\{z_{n}\right\},\left\{x_{n}\right\}$ provided $z_{n} \in \Phi_{n}$, $x_{n} \in \Lambda_{n}\left(z_{n}\right)$ and $x_{n} \notin \Phi_{n}$, for every $n \in \mathbb{N}$, we get

$$
\begin{aligned}
n< & \left\|x_{n}\right\|_{0} \\
\leq & \mathscr{M}_{1}\left\|z_{0}\right\|+\delta_{r}(0)+\mathfrak{M}_{h}+\frac{\mathscr{M}_{1} \mathscr{M}_{2} \mathfrak{M}_{h} q}{\Gamma(1+q)}\left[\left(\frac{1-\kappa_{1}}{q-\kappa_{1}}\right) b^{\frac{q-\kappa_{1}}{1-\kappa_{1}}}\right]^{1-\kappa_{1}} \\
& +\frac{\mathscr{M}_{1} q}{\Gamma(1+q)}\left[\left(\frac{1-\kappa_{1}}{q-\kappa_{1}}\right) b^{\frac{q-\kappa_{1}}{1-\kappa_{1}}}\right]^{1-\kappa_{1}} \int_{0}^{b}|\rho(\xi)|^{\frac{1}{\kappa_{1}}}\left(1+\left\|z_{n}(\xi)\right\|^{\frac{1}{\kappa_{1}}} d \xi\right)^{\kappa_{1}} \\
\leq & \mathscr{M}_{1}\left\|z_{0}\right\|+\delta_{r}(0)+\mathfrak{M}_{h} \\
& +\frac{\mathscr{M}_{1} q}{\Gamma(1+q)}\left[\left(\frac{1-\kappa_{1}}{q-\kappa_{1}}\right) b^{\frac{q-\kappa_{1}}{1-\kappa_{1}}}\right]^{1-\kappa_{1}}\left(\mathscr{M}_{2} \mathfrak{M}_{h}+(1+n)\|\rho\|_{\frac{1}{\kappa_{1}}}\right), \quad n \in \mathbb{N},
\end{aligned}
$$

which contradicts (3.2). The conclusion refers to Theorem 2.1, like Theorem 3.1.

Theorem 3.3 Assuming that $\left(\mathbf{H}_{\mathbf{1}}\right)-\left(\mathbf{H}_{5}\right)$ holds. $\left(\mathbf{H}_{\mathbf{9}}\right)$ there exist $\beta \in L^{\frac{1}{\kappa_{1}}}\left([0, b] ; \mathbb{R}_{+}\right)$and a nondecreasing function $\varrho:[0, \infty) \rightarrow[0, \infty)$ fulfilling

$$
\|\mathscr{H}(u, d)\| \leq \beta(u) \varrho(\|d\|),
$$

for almost everywhere $u \in[0, b], d \in \mathbb{Y}$, and $\mathscr{L}>0$ provided

$$
\frac{\mathscr{L}}{\mathscr{M}_{1}\left\|z_{0}\right\|+\delta_{r}(0)+\mathfrak{M}_{h}+\frac{\mathscr{M}_{1} q}{\Gamma(1+q)}\left[\left(\frac{1-\kappa_{1}}{q-\kappa_{1}}\right) b^{\frac{q-\kappa_{1}}{1-\kappa_{1}}}\right]^{1-\kappa_{1}}\left(\mathscr{M}_{2} \mathfrak{M}_{h}+\|\rho\|_{\frac{1}{\kappa_{1}}}\right) \varrho(\mathscr{L})}>1
$$

then (1.1)-(1.2) possess at least a mild solution.

Proof We have to ensure that $\Lambda$ maps the ball $\Phi_{\mathscr{L}}$ into itself. For any $z \in \Phi_{\mathscr{L}}, x \in \Gamma(z)$, we conclude

$$
\begin{aligned}
\left\|x_{n}\right\|_{0} \leq & \mathscr{M}_{1}\left\|z_{0}\right\|+\delta_{r}(0)+\mathfrak{M}_{h}+\frac{\mathscr{M}_{1} \mathscr{M}_{2} \mathfrak{M}_{h} q}{\Gamma(1+q)}\left[\left(\frac{1-\kappa_{1}}{q-\kappa_{1}}\right) b^{\frac{q-\kappa_{1}}{1-\kappa_{1}}}\right]^{1-\kappa_{1}} \\
& +\frac{\mathscr{M}_{1} q}{\Gamma(1+q)}\left[\left(\frac{1-\kappa_{1}}{q-\kappa_{1}}\right) b^{\frac{q-\kappa_{1}}{1-\kappa_{1}}}\right]^{1-\kappa_{1}} \int_{0}^{b}|\rho(\xi)|^{\frac{1}{\kappa_{1}}}\left(\varrho\|z(\xi)\|^{\frac{1}{\kappa_{1}}} d \xi\right)^{\kappa_{1}} \\
\leq & \mathscr{M}_{1}\left\|z_{0}\right\|+\delta_{r}(0)+\mathfrak{M}_{h} \\
& +\frac{\mathscr{M}_{1} q}{\Gamma(1+q)}\left[\left(\frac{1-\kappa_{1}}{q-\kappa_{1}}\right) b^{\frac{q-\kappa_{1}}{1-\kappa_{1}}}\right]^{1-\kappa_{1}}\left[\mathscr{M}_{2} \mathfrak{M}_{h}+\|\beta\|_{\frac{1}{\kappa_{1}}}\right] \varrho(\mathscr{L})<\mathscr{L} .
\end{aligned}
$$

This indicates that (1.1)-(1.2) possess at least a mild solution.

\section{Nonlocal conditions}

The active desire for analyzing fractional systems with nonlocal problems comes mainly from theoretical physics. The outcomes regarding the existence of Cauchy problems using nonlocal conditions were firstly investigated by Byszewski [20]. Many papers [13, 21, 34, 35,53 ] have acknowledged the facts of the existence, controllability and uniqueness for 
varied nonlinear fractional systems and abstract differential systems. Motivated by the above discussions, this part deals with the existence of (1.1)-(1.2) as

$$
\begin{aligned}
& { }^{\mathrm{C}} D_{t}^{q}[z(u)-h(u, z(u))] \in \mathscr{A} z(u)+\mathscr{H}(u, z(u)), \quad u \in[0, b], \\
& z(0)+\varphi(z)=z_{0}
\end{aligned}
$$

where $\varphi: \mathfrak{C}([0, b] ; \mathbb{Y}) \rightarrow \mathbb{Y}$ fulfills the following conditions:

$\left(\mathbf{H}_{10}\right)$ For some constant $\mathscr{N}>0$ provided $\|\varphi(z)\| \leq \mathscr{N}, z \in \mathfrak{C}([0, b] ; \mathbb{Y})$.

$\left(\mathbf{H}_{11}\right)$ There is a constant $\mathscr{L}>0$ and

$$
\frac{\mathscr{L}}{\mathscr{M}_{1}\left\|z_{0}\right\|+\mathscr{N}+\delta_{r}(0)+\mathfrak{M}_{h}+\frac{\mathscr{M}_{1} q}{\Gamma(1+q)}\left[\left(\frac{1-\kappa_{1}}{q-\kappa_{1}}\right) b^{\frac{q-\kappa_{1}}{1-\kappa_{1}}}\right]^{1-\kappa_{1}}\left(\mathscr{M}_{2} \mathfrak{M}_{h}+\|\rho\|_{\frac{1}{\kappa_{1}}}\right) \varrho(\mathscr{L})}
$$$$
>1 \text {. }
$$

In order to show the high accuracy, we always refer the nonlocal condition in the place of initial condition $z(0)=z_{0}$. Particularly, $\varphi(z)$ can be formulated as

$$
\varphi(z)=\sum_{i=1}^{n} K_{i} z\left(t_{i}\right)
$$

where $K_{i}(i=1,2,3, \ldots, n)$ are constants and $0<t_{1}<t_{2}<\cdots<t_{n} \leq b$.

Definition 4.1 $z:[0, b] \rightarrow \mathbb{Y}$ is called the mild solution of the neutral fractional differential model (4.1)-(4.2) if the accompanying recognize $z(0)+\varphi(z)=z_{0}$ and there exists $\chi \in L^{\frac{1}{\kappa_{1}}}([0, b] ; \mathbb{Y})$ provided $\chi(u) \in \mathscr{H}(u, z(u))$ on $u \in[0, b]$ and $z$ satisfies

$$
\begin{aligned}
z(u)= & \mathbb{T}(u)\left[z_{0}-\varphi(z)-h\left(0, z_{0}\right)\right]+h(u, z(u))+\int_{0}^{u}(u-w)^{q-1} \mathscr{A} \mathbb{S}(u-w) h(w, z(w)) d w \\
& +\int_{0}^{u}(u-w)^{q-1} \mathbb{S}(u-w) \chi(w) d w, \quad u \in[0, b],
\end{aligned}
$$

such that $\mathbb{T}(u)$ and $\mathbb{S}(u)$ are defined as in Definition 3.1.

Theorem 4.1 If Theorem 3.1, Theorem 3.2 and Theorem 3.3 hold, and in addition hypotheses $\left(\mathbf{H}_{10}\right)$ and $\left(\mathbf{H}_{11}\right)$ hold, then the neutral fractional system with inclusion (4.1)-(4.2) has at least a mild solution.

Proof We introduce the solution operator $\Lambda: \mathfrak{C}([0, b] ; \mathbb{Y}) \multimap \mathfrak{C}([0, b] ; \mathbb{Y})$ as

$$
\begin{aligned}
\Lambda(\wp)= & \left\{z \in \mathfrak{C}([0, b] ; \mathbb{Y}): z(u)=\mathbb{T}\left(z_{0}-\varphi(z)-h\left(0, z_{0}\right)\right)+h(u, \wp(u))\right. \\
& \left.+S_{1}(z)(u)+S_{2}(\chi)(u)\right\} .
\end{aligned}
$$

It should be noted that we recognize that $\Lambda$ possesses a fixed point by employing the techniques utilized in Theorems corresponding to initial conditions. The proof is similar, therefore we omitted it. 


\section{An example}

Let us consider the model:

$$
\begin{aligned}
& { }^{\mathrm{C}} D_{u}^{q}\left[v(u, y)+\int_{0}^{\pi} k(\theta, y) v(u, \theta) d \theta\right] \in \frac{\partial^{2}}{\partial y^{2}} v(u, y)+s(u, v(u, y)), \quad u \in I=[0, \pi], \\
& v(u, 0)=v(u, \pi)=0, \\
& v(0, y)=0, \quad 0<y<\pi,
\end{aligned}
$$

where $0<q \leq 1$, construct $\mathbb{Y}=L^{2}(0, \pi)$ and $\mathscr{A}: D(\mathscr{A}) \subseteq \mathbb{Y} \rightarrow \mathbb{Y}$ by $\mathscr{A} z=z^{\prime \prime}$, together $D(\mathscr{A})=\left\{z \in \mathbb{Y}: y^{\prime \prime} \in \mathbb{Y}\right\}$, are absolutely continuous. Obviously $\mathscr{A}$ is the infinitesimal generator of $\{T(u), u \geq 0\}$ in $\mathbb{Y}$ and generates the strongly continuous semi group $T(u)$. Additionally, $\mathscr{A}$ has eigenvalues in the form of $-n^{2}, n \in \mathbb{N}$, and it can be denoted as

$$
\mathscr{A} z=\sum_{n=1}^{\infty} n^{2}\left\langle z, z_{n}\right\rangle, \quad z \in D(\mathscr{A})
$$

and $z_{n}(x)=\sqrt{\frac{2}{\pi}} \sin (n x), n=1,2,3, \ldots$, represents the set of eigenvectors of $\mathscr{A}$ which are orthonormal. Also for any $z \in \mathbb{Y}$,

$$
T(u) z=\sum_{n=1}^{\infty} e^{-n^{2}} u\left\langle z, z_{n}\right\rangle z_{n} .
$$

Clearly, $T(u)$ fulfills $\left(\mathbf{H}_{\mathbf{1}}\right)$. Define $h:[0, \pi] \times \mathbb{Y} \rightarrow \mathbb{Y}$ by

$$
h(v)(z)=\int_{0}^{\pi} k(\theta, z) v(u, \theta) d \theta
$$

where the continuous function $k:[0, \pi] \times[0, \pi] \rightarrow \mathbb{R}$ provided $\|k(\cdot, z)\| \leq 1$, for each $z \in[0, \pi]$ and $v(u)(z)=v(u, z), \mathscr{H}(u, z(u)) z=s(u, v(u, z))$. With a suitable choice of $\mathscr{A}, \mathscr{H}$, $h$, the above said system can be equivalent to (1.1)-(1.2), that is,

$$
\begin{aligned}
& { }^{\mathrm{C}} D_{t}^{q}[z(u)-h(u, z(u))] \in \mathscr{A} z(u)+\mathscr{H}(u, z(u)), \quad u \in[0, b], \\
& z(0)+\varphi(z)=z_{0} .
\end{aligned}
$$

Besides assuming $\mathscr{H}, h$ satisfies the concerned hypotheses. As a result, (5.1)-(5.2) has at least a mild solution on $[0, b]$.

\section{Conclusion}

This manuscript addresses the existence of Caputo fractional neutral inclusions without compactness in a Banach space by using weak topology. Further, the results are derived for fractional neutral system where nonlocal conditions hold. Our theorem guarantees the effectiveness of the existence, which is the result of the system concerned. 
Availability of data and materials

Not applicable.

\section{Competing interests}

There does not exist any competing interest regarding this article.

\section{Authors' contributions}

All authors have equally made contributions to this article. All authors read and approved the final manuscript.

\section{Author details}

'Department of Mathematical Sciences, Princess Nourah bint Abdulrahman University, Riyadh, Saudi Arabia. ${ }^{2}$ PG and Research Department of Mathematics, Kongunadu Arts and Science College (Autonomous), Coimbatore, India. ${ }^{3}$ Department of Mathematics and General Sciences, Prince Sultan University, Riyadh, Saudi Arabia. ${ }^{4}$ Department of Medical Research, China Medical University, Taichung, Taiwan. ${ }^{5}$ Department of Computer Science and Information Engineering, Asia University, Taichung, Taiwan. ${ }^{6}$ Department of Mathematics, Sri Eshwar College of Engineering (Autonomous), Coimbatore, India.

\section{Publisher's Note}

Springer Nature remains neutral with regard to jurisdictional claims in published maps and institutional affiliations.

Received: 9 October 2019 Accepted: 9 December 2019 Published online: 19 December 2019

\section{References}

1. Abada, N., Benchohra, M., Hammouche, H.: Existence and controllability results for nondensely defined impulsive semilinear functional differential inclusions. J. Differ. Equ. 246, 3834-3863 (2009)

2. Adjabi, Y., Jarad, F., Baleanu, D., Abdeljawad, T.: On Cauchy problems with Caputo Hadamard fractional derivatives. J. Comput. Anal. Appl. 21, 661-681 (2016)

3. Ali Dokuyucu, M., Celik, E., Bulut, H., Baskonus, H.M.: Cancer treatment model with the Caputo-Fabrizio fractional derivative. Eur. Phys. J. Plus 133, 1-6 (2018)

4. Alzabut, J.O., Abdeljawad, T., Baleanu, D.: Nonlinear delay fractional difference equations with applications on discrete fractional Lotka-Volterra competition model. J. Comput. Anal. Appl. 25, 89-898 (2018)

5. Anastassiou, G.A., Argyros, I.K.: Approximating fixed points with applications in fractional calculus. J. Comput. Anal. Appl. 21, 1225-1242 (2016)

6. Anguraj, A., Kanjanadevi, S., Baleanu, D.: On mild solution of abstract neutral fractional order impulsive differential equations with infinite delay. J. Comput. Anal. Appl. 24, 1232-1244 (2018)

7. Baleanu, D., Gunvenc, Z.B., Tenreiro Machado, J.A.: New Trends in Nanotechnology and Fractional Calculus Applications. Springer, Berlin (2010)

8. Baleanu, D., Mousalou, A., Rezapour, S.: On the existence of solutions for some infinite coefficient-symmetric Caputo-Fabrizio fractional integro-differential equations. Bound. Value Probl. 2017, 145 (2017)

9. Baleanu, D., Mousalou, A., Rezapour, S.: A new method for investigating approximate solutions of some fractional integro-differential equations involving the Caputo-Fabrizio derivative. Adv. Differ. Equ. 2017, 51 (2017)

10. Baleanu, D., Mousalou, A., Rezapour, S.: The extended fractional Caputo-Fabrizio derivative of order $0 \leq \sigma<1$ on $C_{\mathbb{R}}[0,1]$ and the existence of solutions for two higher-order series-type differential equations. Adv. Differ. Equ. 2018, 255 (2018)

11. Baleanu, D., Rezapour, S., Saberpour, Z:: On fractional integro-differential inclusions via the extended fractional Caputo-Fabrizio derivation. Bound. Value Probl. 2019, 79 (2019)

12. Baleanu, D., Shiri, B.: Collocation methods for fractional differential equations involving non-singular kernel. Chaos Solitons Fractals 116, 136-145 (2018)

13. Benchohra, M., Gatsori, E.P., Ntouyas, S.K.: Controllability results for semilinear evolution inclusions with nonlocal conditions. J. Optim. Theory Appl. 118, 493-513 (2003)

14. Benedetti, I., Malaguti, L., Taddei, V.: Semilinear differential inclusions via weak topologies. J. Math. Anal. Appl. 368 90-102 (2010)

15. Benedetti, I., Obukhovskii, V., Taddei, V.: Controllability for systems governed by semilinear evolution inclusion without compactness. Nonlinear Differ. Equ. Appl. 21, 795-812 (2014)

16. Benedetti, I., Obukhovskii, V., Zecca, P.: Controllability for impulsive semilinear differential inclusions in a Banach space with a non-compact semigroup. Discuss. Math., Differ. Incl. Control Optim. 31, 39-69 (2011)

17. Benedetti, I., Rubbioni, P.: Existence of solutions on compact and non-compact intervals for semilinear impulsive differential inclusions with delay. Topol. Methods Nonlinear Anal. 32, 227-245 (2008)

18. Bochner, S., Taylor, A.E.: Linear functionals on certain spaces of abstractly valued functions. Ann. Math. 39, 913-944 (1938)

19. Brezis, H.: Analyse Foctionelle, Théorie et Applications. Masson, Paris (1983)

20. Byszewski, L.: Theorems about the existence and uniqueness of solutions of a semilinear evolution nonlocal Cauchy problem. J. Math. Anal. Appl. 162, 494-505 (1991)

21. Chang, Y.K., Nieto, J.J.: Existence of solutions for impulsive neutral integro-differential inclusions with nonlocal initial conditions via fractional operators. Numer. Funct. Anal. Optim. 30, 227-244 (2009)

22. Debbouche, A., Baleanu, D.: Controllability of fractional evolution nonlocal impulsive quasilinear delay integro-differential systems. Comput. Math. Appl. 62, 1442-1450 (2011)

23. Dunford, N., Schwartz, J.T.: Linear Operators. Wiley, New York (1998)

24. El-Sayed, A.M.A., Ibrahim, A.G.: Set-valued integral equations of arbitrary (fractional) order. Appl. Math. Comput. 118, 113-121 (2001) 
25. Gambo, Y.Y., Ameen, R., Jarad, F., Abdeljawad, T.: Existence and uniqueness of solutions to fractional differential equations in the frame of generalized Caputo fractional derivatives. Adv. Differ. Equ. 2018, 134 (2018)

26. Jarad, F., Abdeljawad, T., Baleanu, D.: On the generalized fractional derivatives and their Caputo modification. J. Nonlinear Sci. Appl. 10, 2607-2619 (2017)

27. Jothimani, K., Kaliraj, K., Hammouch, Z., Ravichandran, C.: New results on controllability in the framework of fractional integro-differential equations with nondense domain. Eur. Phys. J. Plus 134, 441 (2019)

28. Jothimani, K., Valliammal, N., Ravichandran, C.: Existence result for a neutral fractional integro-differential equation with state dependent delay. J. Appl. Nonlinear Dyn. 7, 371-381 (2018)

29. Kantorovich, L.V., Akilov, G.P.: Functional Analysis. Pergamon, Oxford (1982)

30. Khan, H., Khan, A., Abdeljawad, T., Alkhazzan, A.: Existence results in Banach space for a nonlinear impulsive system. Adv. Differ. Equ. 2019, 18 (2019)

31. Kilbas, A.A., Srivastava, H.M., Trujillo, J.J.: Theory and Applications of Fractional Differential Equations. North-Holland Mathematics Studies. Elsevier, Amsterdam (2006)

32. Lakshmikantham, V., Leela, S., Vasundhara Devi, J.: Theory of Fractional Dynamic Systems. Cambridge Scientific Publishers, Cambridge (2009)

33. Li, Y.: Controllability of nonlinear neutral fractional impulsive differential inclusions in Banach spaces. Adv. Differ. Equ. 2014, 234 (2014)

34. Liu, H., Liao, C.W., Pang, C.T.: Existence of some semilinear nonlocal functional differential equations of neutral type. Abstr. Appl. Anal. 2013, Article ID 503656 (2013)

35. Machado, J.A., Ravichandran, C., Rivero, M., Trujillo, J.J.: Controllability results for impulsive mixed-type functional integro-differential evolution equations with nonlocal conditions. Fixed Point Theory Appl. 2013, 66 (2013)

36. Melike Aydogan, S., Baleanu, D., Mousalou, A., Rezapour, S.: On approximate solutions for two higher-order Caputo-Fabrizio fractional integro-differential equations. Adv. Differ. Equ. 2017, 221 (2017)

37. Melike Aydogan, S., Baleanu, D., Mousalou, A., Rezapour, S.: On high order fractional integro-differential equations including the Caputo-Fabrizio derivative. Bound. Value Probl. 2018, 90 (2018)

38. Miller, K.S., Ross, B.: An Introduction to the Fractional Calculus and Differential Equations. Wiley, New York (1993)

39. O'Regan, D.: Fixed point theorems for weakly sequentially closed maps. Trans. Am. Math. Soc. 44, $277-304$ (1938)

40. Pazy, A.: Semigroups of Linear Operators and Applications to Partial Differential Equations. Springer, New York (1983)

41. Pettis, B.J.: On the integration in vector spaces. Arch. Math. 36, 61-70 (2000)

42. Podlubny, I.: Fractional Differential Equations. An Introduction to Fractional Derivatives, Fractional Differential Equations, to Methods of Their Solution and Some of Their Applications. Academic Press, San Diego (1999)

43. Ravichandran, C., Baleanu, D.: Existence results for fractional functional integro-differential evolution equations with infinite delay in Banach spaces. Adv. Differ. Equ. 2013, 215 (2013)

44. Ravichandran, C., Baleanu, D.: On the controllability of fractional functional integro-differential systems with infinite delay in a Banach spaces. Adv. Differ. Equ. 2013, 291 (2013)

45. Ravichandran, C., Jothimani, K., Baskonus, H.M., Valliammal, N.: New results on nondensely characterized integro-differential equations with fractional order. Eur. Phys. J. Plus 133, 109 (2018)

46. Ravichandran, C., Trujillo, J.J.: Controllability of impulsive fractional functional integro-differential equations in Banach spaces. J. Funct. Spaces 2013, Article ID 812501 (2013)

47. Ravichandran, C., Valliammal, N., Nieto, J.J.: New results on exact controllability of a class of fractional neutral integro-differential systems with state-dependent delay in Banach spaces. J. Franklin Inst. 356, 1535-1565 (2019)

48. Shiri, B., Baleanu, D.: System of fractional differential algebraic equations with applications. Chaos Solitons Fractals 120, 203-212 (2019)

49. Valliammal, N., Ravichandran, C.: Results on fractional neutral integro-differential systems with state-dependent delay in Banach spaces. Nonlinear Stud. 25, 1-13 (2018)

50. Valliammal, N., Ravichandran, C., Park, J.H.: On the controllability of fractional neutral integro-differential delay equations with nonlocal conditions. Math. Methods Appl. Sci. 40, 5044-5055 (2017)

51. Vijayakumar, V.: Approximate controllability results for non-densely defined fractional neutral differential inclusions with Hille-Yosida operators. Int. J. Control 92, 2210-2222 (2019)

52. Vijayakumar, V., Murugesu, R.: Controllability for a class of second-order evolution differential inclusions without compactness. Appl. Anal. 98, 1367-1385 (2019)

53. Vijayakumar, V., Ravichandran, C., Murugesu, R.: Nonlocal controllability of mixed Volterra-Fredholm type fractional semilinear integro-differential inclusions in Banach spaces. Dyn. Contin. Discrete Impuls. Syst. 20, 485-502 (2013)

54. Vijayakumar, V., Ravichandran, C., Murugesu, R.: Approximate controllability for a class of fractional neutral integro-differential inclusions with state-dependent delay. Nonlinear Stud. 20, 511-530 (2013)

55. Wang, J., Zhou, Y.: Existence and controllability results for fractional semilinear differential inclusions. Nonlinear Anal., Real World Appl. 12, 3642-3653 (2011)

56. Zhou, Y.: Basic Theory of Fractional Differential Equations. World Scientific, Singapore (2014)

57. Zhou, Y.: Fractional Evolution Equations and Inclusions, Analysis and Control. Elsevier/Academic Press, London (2016)

58. Zhou, Y.: Attractivity for fractional differential equations in Banach space. Appl. Math. Lett. (2017). https://doi.org/10.1016/j.aml.2017.06.008

59. Zhou, Y., Vijayakumar, V., Murugesu, R.: Controllability for fractional evolution inclusions without compactness. Evol. Equ. Control Theory 4, 507-524 (2015) 